Provided for non-commercial research and education use. Not for reproduction, distribution or commercial use.
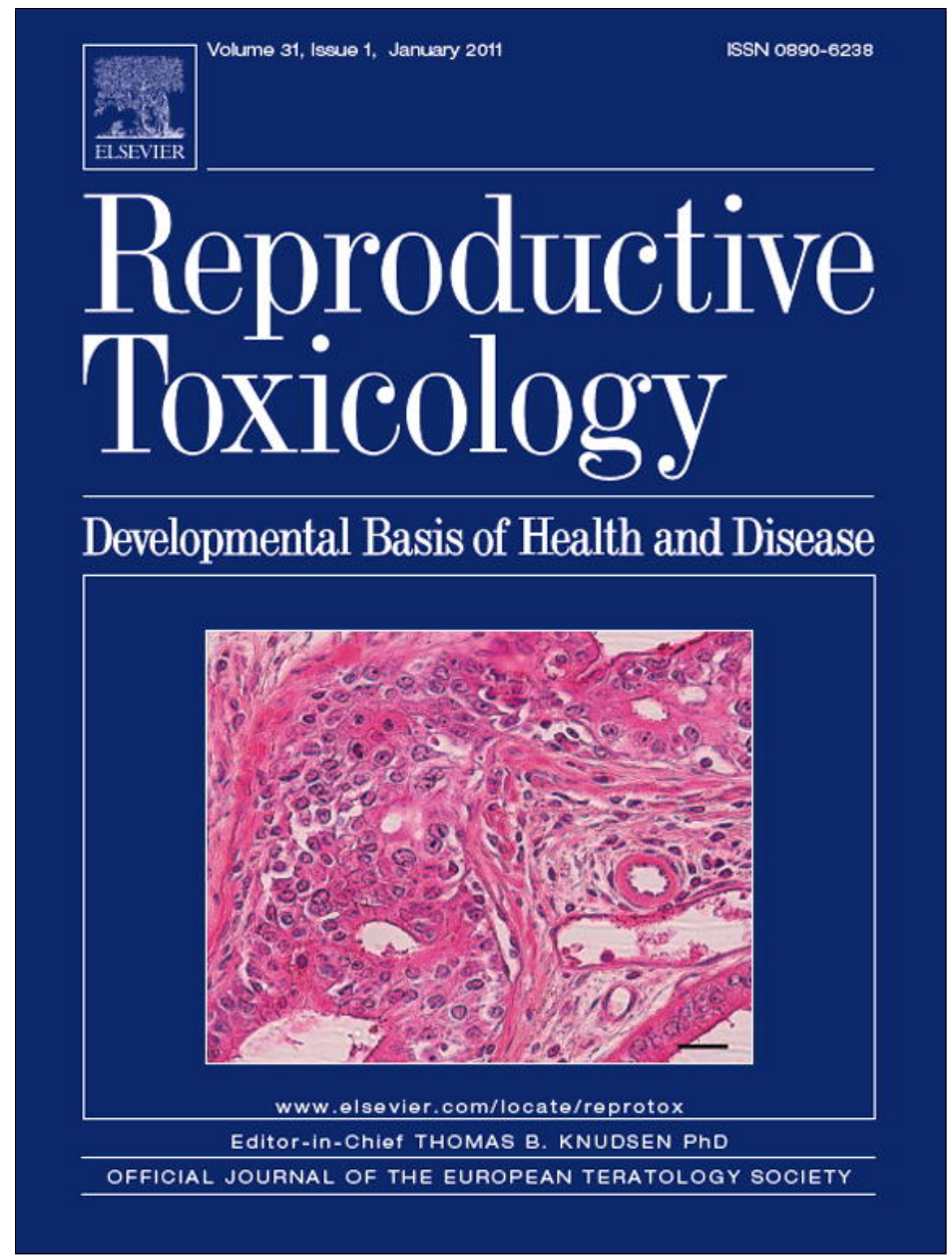

This article appeared in a journal published by Elsevier. The attached copy is furnished to the author for internal non-commercial research and education use, including for instruction at the authors institution and sharing with colleagues.

Other uses, including reproduction and distribution, or selling or licensing copies, or posting to personal, institutional or third party websites are prohibited.

In most cases authors are permitted to post their version of the article (e.g. in Word or Tex form) to their personal website or institutional repository. Authors requiring further information regarding Elsevier's archiving and manuscript policies are encouraged to visit:

http://www.elsevier.com/copyright 


\title{
Differential effects of p,p'-DDE on testis and liver mitochondria:Implications for reproductive toxicology
}

\author{
Paula C. Mota ${ }^{a}$, Marília Cordeiro ${ }^{a}$, Susana P. Pereira ${ }^{a}$, Paulo J. Oliveira ${ }^{a}$, \\ António J. Moreno ${ }^{\mathrm{b}}$, João Ramalho-Santos ${ }^{\mathrm{a}, *}$ \\ a Center for Neuroscience and Cell Biology, Department of Life Sciences, University of Coimbra, Portugal \\ b Institute for Marine Research, Department of Life Sciences, University of Coimbra, Portugal
}

\section{A R T I C L E I N F O}

\section{Article history:}

Received 28 July 2010

Received in revised form 25 August 2010

Accepted 10 September 2010

Available online 15 October 2010

\section{Keywords:}

2,2-Bis(4-chlorophenyl)-1,1-dichloro-

ethylene

(DDE)

Testis

Mitochondria

Bioenergetics

Male fertility

\begin{abstract}
A B S T R A C T
The release of environmental contaminants can contribute to impaired male fertility. The bioenergetics of isolated liver mitochondria have been used as a toxicological indicator, an inexpensive first line model to screen possible effects of several substances. Here we report the effects of 2,2-bis(4-chlorophenyl)1,1-dichloro-ethylene (DDE) on the bioenergetical parameters of testicular mitochondria. A significant decrease in repolarization potential (after a phosphorylative cycle), state 3 respiration and uncoupled respiration, with a concomitant increase in lag phase was found, demonstrating a decrease in mitochondrial function. Importantly, there was also a clear increase in maximum potential in DDE-treated testis mitochondria, which was not mirrored by more commonly used liver mitochondria. Indeed, comparative studies showed that testis and liver mitochondria have strikingly different sensitivities and patterns of response to DDE, indicating that testis mitochondria should be used as a primary toxicological model for a proper evaluation of putative effects of environmental toxicants on the bioenergetics of spermatogenesis and male fertility.

(c) 2010 Elsevier Inc. All rights reserved.
\end{abstract}

\section{Introduction}

Environmental contaminants have a deleterious effect on reproductive function, either by directly affecting germ cells, including damage to sperm nuclear DNA and increased ROS production, or by mimicking hormonal action [1,2]. These effects may be transgenerational, probably through changes in methylation patterns [3]. 1,1-Dichlorodiphenyltrichloroethane (DDT) and its metabolite, 2,2-bis(4-chlorophenyl)-1,1-dichloro-ethylene (DDE), are environmental contaminants present in all living organisms. Due to their lipophylic nature, resistance to degradation and food chain bioaccumulation, high concentrations have been reported in tissues, even in animals from remote areas [4]. The first studies on reproductive changes induced by DDT and its metabolite DDE were performed in birds, where they were identified as a cause of egg shell thinning [5], followed by reported interactions with the

Abbreviations: DDE, 2,2-bis(4-chlorophenyl)-1,1-dichloro-ethylene; DDT, 1,1dichlorodiphenyltrichloroethane; ROS, reactive oxygen species; SOD, superoxide dismutases; GSH-Px, glutathione peroxidases; DMSO, dimethyl sulfoxide; RCR respiratory control ratio; HPLC, reverse-phase high performance liquid chromatography; $\Delta \psi$, mitochondrial transmembrane electrical potential; TPP $^{+}$, tetraphenyl phosphonium.

* Corresponding author. Tel.: +351 239855760.

E-mail address: jramalho@ci.uc.pt (J. Ramalho-Santos). estrogen receptor in alligators [6]. Using a rat model DDE was described as antagonizing the androgen receptor leading to a slew of effects, from gonadal development to accessory gland weight [7]. In recent studies DDE was shown to affect Sertoli cells in culture, decreasing the expression of transferrin and increasing the expression of androgen-binding protein [8], similarly to other known anti-androgen compounds. Furthermore death of Sertoli cells by apoptosis through the mitochondrial pathway, and an increase in ROS production dependent on DDE concentration used, was also observed [9], and in pre-pubertal rats DDE induced a significant decrease in SOD and GSH-Px activity. Lipid peroxidation was also increased confirming oxidative stress as an important component in DDE-induced toxicity [10].

It has been shown that many drugs have deleterious effects on mitochondrial function and that mitochondria are one of the primary targets for toxic injury, leading to dysfunction due to energy imbalance, excessive ROS production and/or apoptosis. In this context, mitochondrial studies have revealed toxicological and pharmacologically relevant mechanisms for xenobiotic activity. These studies have mostly been carried out using liver mitochondria, giving the importance of this organ in metabolism and detoxification of foreign substances (reviewed by [11]). Such a model system showed that DDE caused inhibition of the mitochondrial enzymes NADH-Ubiquinone oxireductase and succinate dehydrogenase, together with an increase of mitochondrial mem- 
brane proton leak and decreased transport of inorganic phosphate, which may lead to a deficit in the energy level of hepatocytes [12]. However previous studies of testicular bioenergetics both in rat [13] and domestic cat (Felis catus) [14] have demonstrated major differences in bioenergetic parameters between liver and testicular mitochondria under physiological conditions, especially when measuring state 3 and 4 respiration and RCR, using the same protocols [14]. These differences imply a different response of these organelles to drugs, particularly DDE, suggesting that the same substance may affect the bioenergetics of different organs, and thus their functions, differently.

The hypothesis of the present work was that the reported effects of DDE on the male gonad (and thus on the bioenergetics of spermatogenesis) could also involve mitochondrial dysfunction in that tissue, and that these effects may be distinct from those found in the more currently used liver mitochondria. To address this hypothesis, bioenergetic parameters of isolated testis and liver mitochondria from Wistar-Han rats were characterized and compared.

\section{Materials and methods}

\subsection{Materials}

All chemicals were obtained from Sigma, St. Louis, MO, USA, unless otherwise described. DDE (99.1\% de purity) was dissolved in dimethyl sulfoxide (DMSO) to a final concentration of $62.8 \mathrm{mM}(20 \mathrm{mg} / \mathrm{ml})$.

Media used: testis mitochondria isolation medium contained $250 \mathrm{mM}$ sucrose, $0.2 \mathrm{mM}$ EGTA, 0.1 mM EDTA, $5 \mathrm{mM}$ HEPES-KOH ( $\mathrm{pH} 7.4$ ) and $0.1 \%$ defatted BSA (bovine serum albumin). Washing medium was composed by $250 \mathrm{mM}$ sucrose, $5 \mathrm{mM}$ HEPES-KOH ( $\mathrm{pH}$ 7.4). The reaction medium consisted of $65 \mathrm{mM} \mathrm{KCl}, 125 \mathrm{mM}$ sucrose, $10 \mathrm{mM}$ Tris, $20 \mu \mathrm{M}$ EGTA, $2.5 \mathrm{mM} \mathrm{KH} \mathrm{PO}_{4}(\mathrm{pH} 7.4)$ and BSA $(10 \mathrm{~g} / \mathrm{ml})$. Liver mitochondria isolation medium was composed by $300 \mathrm{mM}$ sucrose, $1 \mathrm{mM}$ EGTA, $10 \mathrm{mM}$ HEPES ( $\mathrm{pH}$ 7.4) and $0.1 \%$ defatted BSA. Washing medium was equal to the isolation medium but omitting EGTA and BSA. Liver reaction medium was prepared with $130 \mathrm{mM}$ sacarose, $50 \mathrm{mM} \mathrm{KCl}, 5 \mathrm{mM}$ HEPES, $100 \mathrm{M} \mathrm{TA}, 5 \mathrm{mM} \mathrm{KH}_{2} \mathrm{PO}_{4}$ and $2.5 \mathrm{mM}$ $\mathrm{MgCl}_{2}$.

\subsection{Animals}

Mature male Wistar-Han rats (9-16 weeks) were maintained at the Center for Neuroscience and Cell Biology and Medical School animal facility under controlled light ( $12 \mathrm{~h}$ light $/ 12 \mathrm{~h}$ dark), humidity (45-65\%) and temperature conditions $\left(22-24{ }^{\circ} \mathrm{C}\right)$, with free access to water and food. The study was processed following the rules of animal care established by the European Regulation for Studies with Vertebrate Animals and internal regulation of the Center for Neuroscience and Cell Biology. The animals were euthanized by cervical dislocation followed by decapitation and the testes removed and kept on ice until further use.

\subsection{Isolation of liver and testis mitochondria}

The protocol for isolation of testicular cells mitochondria was adapted from previously described methods [13]. Briefly, testes were decapsulated and finely minced several times in an ice-cold isolation medium. The minced blood-free tissue was then resuspended in isolation medium and homogenized with a tightly fitted Potter-Elvjjem homogenizer (Teflon:glass pestle). The homogenate was then centrifuged at $750 \times \mathrm{g}$ for $10 \mathrm{~min}$ (Sorvall RC-5C, Plus, SS 34 rotor, $4-8^{\circ} \mathrm{C}$ ). The resulting supernatant was centrifuged at $12,000 \times g$ for $10 \mathrm{~min}$. The pellet (mitochondrial fraction) was resuspended twice in washing medium using a paint brush and repelleted at $12,000 \times \mathrm{g}$ for $10 \mathrm{~min}$. Liver mitochondria were isolated following a previously described protocol [15], that is similar to the testis isolation protocol but with specific isolation and washing medium. Mitochondrial protein content was determined by the biuret method, calibrated with BSA. Mitochondria were kept on ice until use.

\subsection{Mitochondrial transmembrane potential and ATPsynthase activity}

Mitochondrial transmembrane potential $(\Delta \psi)$ was estimated with a tetraphenyl phosphonium-selective $\left(\mathrm{TPP}^{+}\right)$electrode by adding $2 \mu \mathrm{M} \mathrm{TPP}^{+}$to the medium. $\Delta \psi$ was estimated according to the equation of Kamo et al. [16], without correction for the "passive" binding contribution of $\mathrm{TPP}^{+}$to the mitochondrial membranes. A matrix volume of $1.1 \mu \mathrm{L} / \mathrm{mg}$ protein was assumed. The parameters analyzed were maximum transmembrane electrical potential, ADPinduced depolarization (corresponding to depolarization elicited by the addition of ADP), lag phase (interval of time correspondent to ADP phosphorylation) and repolarization potential (steady state electric potential obtained after ADP phosphorylation). DDE was added to mitochondria energized with succinate in order to determine effects on maximum transmembrane electrical potential. The incubation period was determined as the lowest time necessary to observe DDE effects on the electrical potential of testicular mitochondria ( $2 \mathrm{~min})$. The DDE concentration used ( $157 \mathrm{nmol} / \mathrm{mg}$ of protein) was selected after previous in vitro assays monitoring changes on the maximum mitochondrial electrical potential in testicular mitochondria as an end-point for drug effect. Control assays were performed by adding the same amount of vehicle - DMSO to the reaction medium after energizing isolated mitochondria.

Following DDE (or control DMSO) incubation ADP was added to the reaction medium (120 and $180 \mathrm{nmol} / 0.8 \mathrm{mg}$ protein for testis and liver mitochondria, respectively). ATPsynthase activity was determinated simultaneously to mitochondrial transmembrane potential using a pH electrode [17]. Experiments were carried out at $30^{\circ} \mathrm{C}$, in $1.5 \mathrm{ml}$ of ATPsynthase medium (similar to reaction medium but with Tris content reduced to $0.5 \mathrm{mM}$ ) plus $2 \mu \mathrm{M} \mathrm{TPP} P^{+}, 3 \mu \mathrm{M}$ rotenone and $1.2 \mathrm{mg}$ of isolated mitochondria. Calibration with a known amount of $\mathrm{HCl}$ was performed at the end of each experiment.

\subsection{Mitochondrial oxygen consumption}

Oxygen consumption of isolated testis mitochondria was monitored polarographically with a Clark-type oxygen electrode [18] connected to a suitable recorder in a $2 \mathrm{ml}$ thermostated water-jacketed chamber with magnetic stirring, at $30^{\circ} \mathrm{C}$. Mitochondria were suspended at a concentration of $0.8 \mathrm{mg} / \mathrm{ml}$ in the reaction medium already including succinate $(5 \mathrm{mM})$ thus energizing mitochondrial via complexes III and IV. Rotenone $(3 \mu \mathrm{M})$ was also added to inhibit complex I and avoid reverse flow of electrons from complex II to complex I. DDE was added $2 \mathrm{~min}$ after succinate $(5 \mathrm{mM})$ and incubated for another $2 \mathrm{~min}$. ADP ( 120 and $180 \mathrm{nmol} / 0.8 \mathrm{mg}$ protein for testis and liver mitochondria, respectively) was added to induce state 3 respiration. After a stable state 4 respiration was reached, oligomycin $(2 \mu \mathrm{g} / \mathrm{ml})$ was added. Two minutes later FCCP $(1 \mu \mathrm{M})$, a mitochondrial uncoupler, was added to determine maximum oxygen consumption. RCR (respiratory control ratio) and ADP/O ratios were calculated according to Chance and Williams [19].

\subsection{Mitochondrial enzyme activity}

All enzymes activities were determined after 4 freeze-thaw cycles to burst mitochondrial membranes and guarantee access to enzymes. Succinate cytochrome $c$ reductase was assayed spectrophotometrically, using cytochrome $c$ as the electron acceptor. Mitochondria were incubated with succinate $(40 \mathrm{mM})$ or succinate plus DDE $(157 \mathrm{nmol} / \mathrm{mg}$ mitochondrial protein) for $5 \mathrm{~min}$ to activate the enzyme. The resulting mixture was then added to a phosphate buffer together with EDTA $(15 \mathrm{mM})$ and $2 \mathrm{mM}$ oxidized cytochrome $c$. The reaction was followed at $550 \mathrm{~nm}$ for $300 \mathrm{~s}$. Complex III activity was then blocked with antimycin A ( $2 \mathrm{mM})$ confirming the reduction of cytochrome $c$ only from electrons donated by succinate. Cytochrome $c$ oxidase (COX) activity was measured polarographically [20], recording oxygen consumption when ascorbate $(5 \mathrm{mM})$ plus TMPD $(0.25 \mathrm{mM})$ were added. COX activity was inhibited by $\mathrm{NaN}_{3}$ after $2 \mathrm{~min}$. DDE $(157 \mathrm{nmol} / \mathrm{mg}$ mitochondrial protein) was incubated with mitochondria for 2 min before adding ascorbate plus TMPD.

\subsection{Proton leak in energized mitochondria}

Proton leak in energized mitochondria can be driven by passive leak through the mitochondria inner membrane or be driven through electric potential dependent proteins. Proton leak, in control and DDE-treated mitochondria, is determined as the relationship between respiration rate and mitochondrial membrane potential, when the membrane potential is altered by titration with electron transport chain inhibitors. Briefly, oxygen consumption and inner membrane potential were simultaneously recorded using a Clark-type oxygen electrode and a TPP+ electrode, respectively. Testis and liver mitochondria $(0.8 \mathrm{mg})$ were incubated in $1 \mathrm{ml}$ of reaction medium with $2 \mu \mathrm{M} \mathrm{TPP}^{+}, 5 \mathrm{mM}$ succinate, $2 \mu \mathrm{M}$ rotenone, oligomycin $(2 \mu \mathrm{g} / \mathrm{ml})$ and $80 \mathrm{ng} / \mathrm{ml}$ of nigericin (added to abolish $\Delta \mathrm{pH}$ so that the total protomotive force can be equal to $\Delta \psi)[13,21]$.

Oxygen consumption and electric potential were progressively inhibited with sequential additions of malonate up to a total of $2 \mathrm{mM}$ and $4 \mathrm{mM}$ in testis and liver mitochondria, respectively. At the end of each experiment, valinomycin was added to dissipate the $\Delta \psi[13,21]$

\section{8. "Proton leak" and potassium permeability in non-energized mitochondria}

Passive proton permeability of the inner membrane in the presence and absence of DDE ( $157 \mathrm{nmol} / \mathrm{mg}$ mitochondrial protein) was estimated by means of the swelling of non-respiring mitochondria in isosmotic medium with $\mathrm{KCH}_{3} \mathrm{COO}$, $\mathrm{NH}_{4} \mathrm{NO}_{3}$ and $\mathrm{KSCN}[22]$. Isolated mitochondria $(1 \mathrm{mg})$ were incubated at $30^{\circ} \mathrm{C}$ in $2 \mathrm{ml}$ ionic medium constituted by $135 \mathrm{mM}$ of $\mathrm{KCH}_{3} \mathrm{COO}, \mathrm{NH}_{4} \mathrm{NO}_{3}$ of absorbance were measured at $540 \mathrm{~nm}$ in a spectrophotometer Jasco V-560 (Jasco Corporation, Japan), in temperature-controlled chambers.

\subsection{Statistical analysis}

All statistical analyses were performed using SPSS (Statistical Package for the Social Sciences Program), version 16.00, software for Windows (SPSS Inc., Chicago, 
A

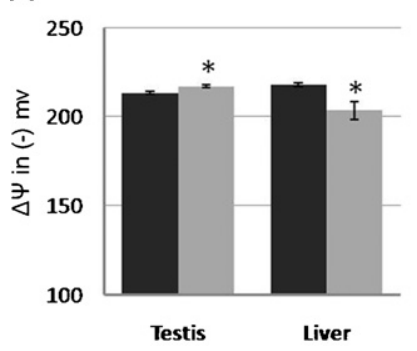

B

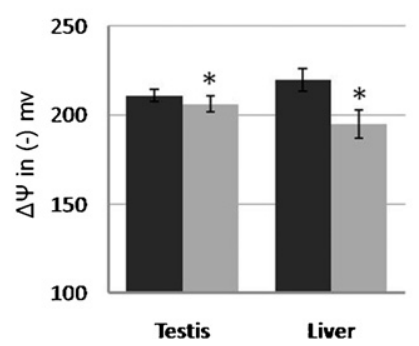

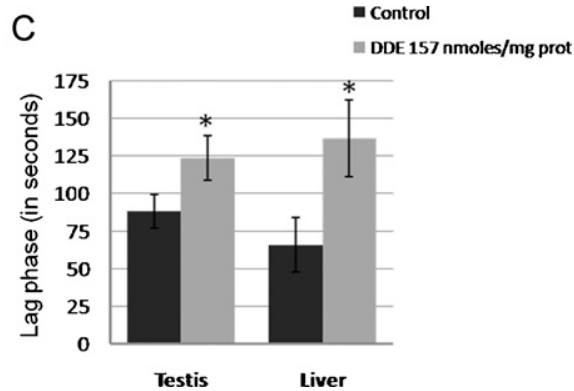

Fig. 1. (A) Maximum electrical potential, (B) repolarization potential and (C) Lag phase were determined using a TPP ${ }^{+}$selective electrode (with an AgCl electrode as reference). Bars represent mean \pm S.E.M. of $n=5$ samples. $\left({ }^{*}\right) P<0.05$ for the paired $T$ test.

IL, USA). All variables were checked for normal distribution. Results are presented as mean \pm S.E.M. of the number of animals indicated. Multiple comparisons were performed using the paired $T$-test. $P<0.05$ was considered significant.

\section{Results}

To better observe possible effects of DDE on mitochondria, ADP concentration was increased in relation to the physiological levels in cells, in order to overload the oxidative phosphorylation system and uncover subtle differences.

A slight increase in maximum electrical potential in isolated testis mitochondria $(P=0.026)$ was observed with DDE, as opposed to liver mitochondria where a significant decrease in the maximum electrical potential developed was obvious $(P=0.003)$ (Fig. 1A). After ADP addition, both testis and liver mitochondria treated with DDE showed increased phosphorylative lag phase and decreased repolarization potential (Fig. 1B), however, in different magnitude as compared to the control. The phosphorylative lag phase increased $40 \%$ in testis mitochondria while liver mitochondria took a double amount of time to phosphorylate the same ADP as control liver mitochondria (Fig. 1C).

ATPsynthase activity recorded at the same time as transmembrane electrical potential, presented similar features, accompanying the increase in lag phase with a significant decrease in activity. However this reduction in activity was not due to a direct effect on complex $\mathrm{V}$, but rather demonstrates the effect of DDE on the respiratory chain. The use of ascorbate plus TMPD as substrates for complex IV revealed no differences on the lag phase and repolarization potential (data not shown) confirming no effect on complex $\mathrm{V}$.

Oxygen consumption revealed further differences between the effects of DDE on testis and liver mitochondria (Table 1). State 3 respiration (oxygen consumption stimulated by ADP phosphorylation) was significantly decreased in both types of mitochondria,

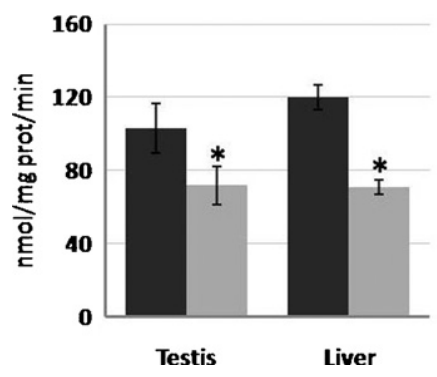

- Control

DDE $157 \mathrm{nmoles} / \mathrm{mg}$ prot

Fig. 3. Succinate cytochrome $c$ reductase activity measured by spectophotometric method using oxidized cytochrome $c$ as an electron acceptor. Bars represent mean \pm S.E.M. of $n=3$ samples $\left({ }^{*}\right) P<0.05$ for the paired $T$ test.

but to a lower extent in testis mitochondria (Fig. 2A). It was when mitochondrial respiration was uncoupled from the phosphorylative system by using FCCP, that the maximum effect of DDE on the respiratory chain could be appreciated, with liver mitochondria presenting an $80 \%$ decrease in uncoupled respiration while testis mitochondria presented a 15\% decrease only (Fig. 2B).

The effects of DDE on the respiratory chain were also explored by directly assaying the activity of enzymes that compose the energy transducing machinery. The activity of succinate-cytochrome $c$ reductase (complexes II and III), showed a significant decrease when testis and liver mitochondria were incubated with DDE $(P=0.005$ and $P=0.002$, respectively); however the effect of DDE on testis succinate-cytochrome $c$ redutase was $15 \%$ less than the one observed in liver mitochondria (Fig. 3). Cytochrome $c$ oxidase presented no differences between control and DDE treated mitochondrial experiments but it is noteworthy to observe that this enzyme presented greater activity in testis mitochondria than in liver mitochondria, contrarily to all other control parameters studied (data not shown).

A

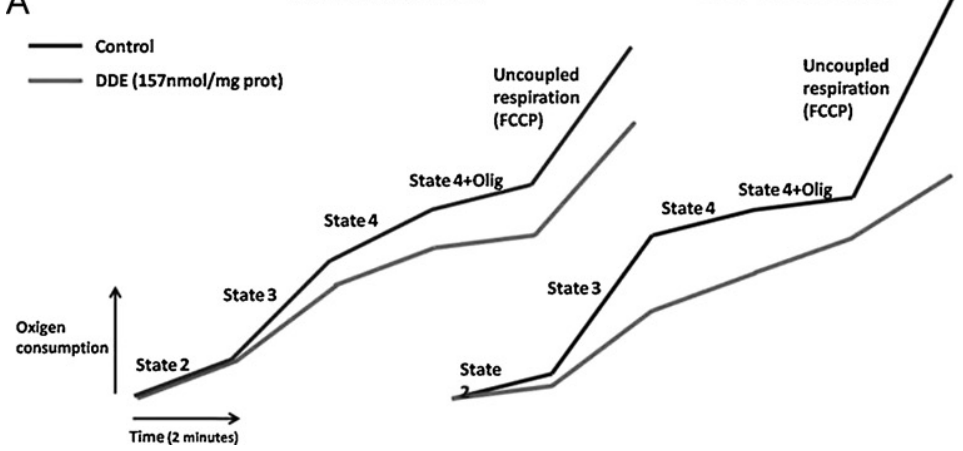

B

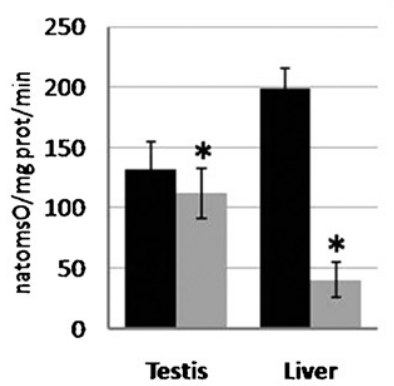

- Control

DDE 157 nmoles/mg prot

Fig. 2. (A) schematic representation of DDE effects observed on oxygen consumption parameters using a Clark-type electrode in testis and liver mitochondria ( $n=10)$. (B) Uncoupled respiration (FCCP $1 \mu \mathrm{M}$ ): bars represent mean \pm S.E.M. of $n=4$ samples treated with FCCP $(1 \mu \mathrm{M})$ to promote mitochondrial uncoupling. $\left({ }^{*}\right) P<0.05$ for the paired $T$ test. 
Table 1

Mitochondrial respiration parameters using succinate as a substrate.

\begin{tabular}{|c|c|c|c|c|c|c|}
\hline & State 3 respiration & State 4 respiration & State oligomycin & Uncoupled respiration & RCR & $\mathrm{ADP} / \mathrm{O}$ \\
\hline Testis control & $93.95 \pm 18.01$ & $46.45 \pm 7.66$ & $21.30 \pm 3.49$ & $131.78 \pm 22.96$ & $2.02 \pm 0.12$ & $1.58 \pm 0.20$ \\
\hline Testis DDE ( $157 \mathrm{nmol} / \mathrm{mg}$ prot $)$ & $71.52 \pm 12.51^{\#}$ & $37.93 \pm 7.10^{\#}$ & $12.21 \pm 1.11^{\#}$ & $112.05 \pm 20.96^{*}$ & $1.93 \pm 0.23$ & $1.44 \pm 0.10^{\ddagger}$ \\
\hline Liver control & $130.82 \pm 17.75$ & $20.38 \pm 2.67$ & $14.95 \pm 2.04$ & $198.71 \pm 17.33$ & $6.46 \pm 1.02$ & $1.95 \pm 0.14$ \\
\hline Liver DDE (157 nmol/mg prot) & $74.89 \pm 18.29^{\#}$ & $27.32 \pm 7.19$ & $27.47 \pm 3.99 *$ & $40.29 \pm 14.27^{*}$ & $3.35 \pm 1.59^{*}$ & $1.98 \pm 0.09$ \\
\hline
\end{tabular}

Oxygen consumption is expressed as natomsO/mg of mitochondrial protein $/ \mathrm{min}$.

* and \# represent $P<0.05$ and $P<0.01$ for the paired $T$ test, respectively. $n=9$ for all parameters except state oligomycin and uncoupled respiration with $n=4$.

State oligomycin, is determined by adding oligomycin to the mitochondria in state 4 respiration, blocking passive leak of protons through ATPsynthase (complex V) and any ATP cycling that may exist (for review see [23]). Any increment in oxygen consumption in this state will be due to an increase in proton leak [24]. Liver mitochondria presented a significant increase $(P=0.049)$ in oxygen consumption in state oligomycin upon DDE treatment indicating an increase in proton leak while testis mitochondria presented a significant decrease $(P=0.008)$, reflecting inhibition of the redox chain or possible addition of this effect with a decrease in proton leak (Table 1).

Given these results, proton leak was further tested through two specific assays; one in energized mitochondria and the second in non-energized mitochondria. Proton leak assayed by titration with malonate in energized liver mitochondria showed that DDEtreated mitochondria presented significantly higher $(P=0.000)$ oxygen consumption for the same transmembrane electrical potential (Fig. 4A) suggesting higher proton leak, while in non-energized mitochondria no differences were found (Fig. 4B). Testis mitochondria treated with DDE presented the same passive proton leak as the control when mitochondria were energized and a significantly less when resting mitochondria were incubated either in isosmotic $\mathrm{KCH}_{3} \mathrm{COO}$ or $\mathrm{NH}_{4} \mathrm{NO}_{3}$ medium. The osmotic swelling assay in KSCN medium also showed some differences between liver and testis mitochondria regarding potassium flux through the mitochondrial membranes. DDE-treated testis mitochondria presented a significant decrease $(P=0.002)$ regarding swelling in relation to control experiments, while in liver mitochondria no differences were observed (Fig. 5).

\section{Discussion}

Previous studies have shown that DDE acts as an endocrine disruptor, namely as a potent androgen antagonist (similar to hydroxyflutamide), interfering with pre-natal development, puberty onset and accessory glands function in adult rats [7], with sex ratios in reptiles and fish and thinning of the egg-shell in birds [25]. However, a few studies using cultured Sertoli cells and in vivo experiments [8-10] suggested that mitochondria might play a role on DDE reproductive pathology.

The protocol used in this work results in a mix of both somatic and germ cell mitochondria. However, given ongoing active spermatogenesis, the percentage of mitochondria derived from the latter is predominant, especially from spermatocytes and spermatids, increasing the number of intermediate and condensed mitochondria present in the isolated fraction [26].

To discard confounding factors, such as the preferential metabolism of DDE in the liver or accumulation in other tissues (fat), we decided to perform an in vitro assay using isolated testis and liver mitochondria, assayed using similar protocols and tested with the same lot of DDE. The minimum dosage of DDE which had a clear effect on testicular mitochondria was used, and this concentration is on the range of levels detected in the wild. In a previous study contamination of fat and liver tissue ranged from a mean of $274.13 \mathrm{mg} / \mathrm{kg}$ wet weight in bird-eating birds to $4.91 \mathrm{mg} / \mathrm{kg}$
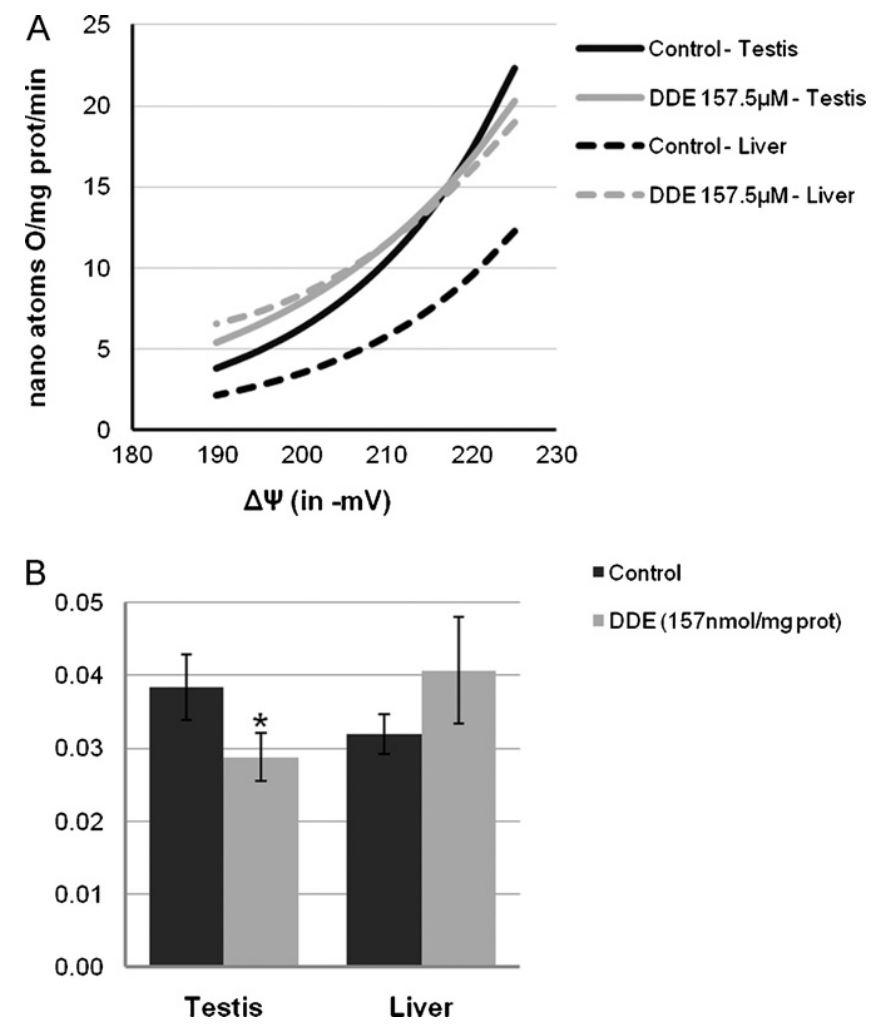

Fig. 4. (A) Proton leak (in energized mitochondria) was determined in 6 separate isolated mitochondria preparations. The data were plotted in dispersion (XY) graph and the best fit model (curve equation) was determined using SPSS 16.0 software. The graph represents values of oxygen consumption, for a given electrical potential, estimated using the curve equation. (B) Osmotic swelling (in non-energized mitochondria). The positive control was determined with FCCP $1 \mu \mathrm{M}$ that promotes $\mathrm{H}^{+}$ leak trough the inner membrane originating the formation of $\mathrm{NH}_{4} \mathrm{NO}_{3}$ in the matrix that drives water entry, mitochondrial swelling and decrease in turbidity (apparent absorbance). $n=3$.

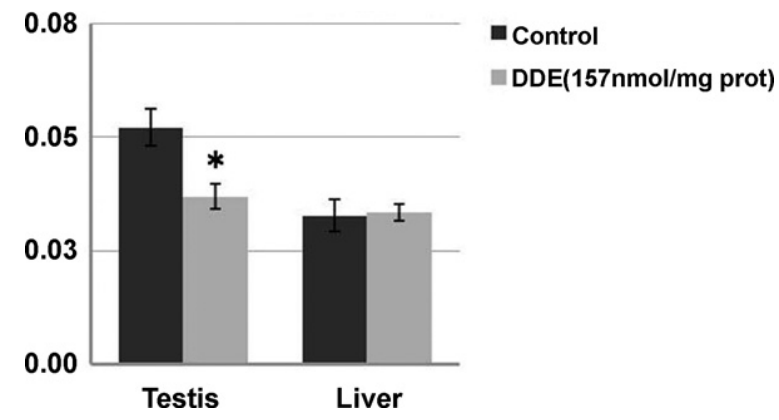

Fig. 5. Permeability to potassium (in non-energized mitochondria). The mitochondria were resuspended in an isosmotic KSCN medium. The anion $\mathrm{SCN}^{-}$crosses the mitochondrial membrane freely and accumulates in the matrix. If the permeability to $\mathrm{K}^{+}$is altered, the KSCN formation in the matrix will osmotically drive water in causing mitochondrial swelling. Bars represent mean \pm S.E.M. of $n=3$ samples. $\left(^{*}\right)$ $P<0.05$ for the paired $T$ test. 
wet weight in insectivorous mammals [27]. Converting the amount of DDE used in our in vitro assay for $\mathrm{mg} / \mathrm{kg}$ wet weight, through mitochondrial isolation yield, which is approximately $10-12 \mathrm{mg}$ of mitochondrial protein for $3.25 \mathrm{~g}$ of testis tissue, we determined that we were using a mean concentration of $160 \mathrm{mg} / \mathrm{kg}$ wet testis weight.

Mitochondrial transmembrane electrical potential was measured with a very sensitive method, which is capable of detecting minor differences, such as the ones invoked by addition of DDE to energized testis mitochondria, which are not detected using widespread mitochondrial probes such as JC-1, for example. Taking into account the available literature we were expecting to see a decrease in maximum electrical potential in succinate-energized mitochondria after adding DDE to testis and liver mitochondria $[9,28,12]$, respectively. This was indeed the case for liver mitochondria, but, in complete contradiction, testis mitochondria presented a small yet significant increase in electrical potential after treatment with DDE, with no increase in state 2 respiration. It has been shown that ROS formation is strongly dependent upon the mitochondrial transmembrane electrical potential. Above state 3 even a slight increase in potential gives rise to a large stimulation of mitochondria-generated ROS [29]. Although minor, the hyperpolarization of the mitochondrial membrane (increase in maximum developed electrical potential) detected in DDE-treated testis mitochondria may be responsible for the increase in ROS production observed in testicular cells treated with DDE $[9,10]$. Furthermore it has already been shown that ROS production could be an early event in mitochondrial mediated apoptosis in Sertoli cells [9], and has also been suggested as the responsible factor for the activation of the Fas/FasL apoptotic pathway in spermatocytes of DDE treated animals [10]. There are also reports in human sperm acknowledging a correlation between DDT concentration in the blood plasma and changes in sperm DNA integrity/condensation [30,31] that could be caused by increased ROS levels during spermatogenesis or epididymal transit. In isolated mitochondria ROS produced by the respiratory chain are diluted in the reaction medium, hindering visualization of ROS effects on the bioenergetic parameters of the mitochondria.

After ADP addition to the medium testis and liver mitochondria treated with DDE showed the same trend of decreasing the repolarization potential and increasing the lag phase. Oxygen consumption during state 3 respiration was also affected by the presence of DDE in both types of mitochondria, but to different extents, leading us to speculate that DDE either did not target succinate dehydrogenase in testis mitochondria or that inhibition of this enzyme by DDE was less effective in testis mitochondria. However, the spectophotometric assay revealed a similar level of inhibition of succinate cytochrome $c$ reductase.

The inhibition of the oxidative phosphorylation observed in rat liver and testis mitochondria may lead to a reduction in the available ATP, especially in more metabolically active germ cells, such as meiotic spermatocytes and spermatids undergoing intense cellular remodeling. The decrease in ATP content could impair spermatogenesis, and spermiogenesis in particular, causing increased levels of morphologically abnormal sperm and altered sperm chromatin integrity/condensation. Indeed several reports have shown correlations between DDT and DDE concentrations and these parameters [30-32].

To test the role of proton leak in DDE-induced mitochondrial toxicity, two different assays were used: titration with malonate in energized mitochondria and osmotic swelling of non-energized mitochondria incubated with isosmotic solutions. Testis mitochondria showed no differences in energized mitochondria, but in non-energized mitochondria a decrease in osmotic swelling with DDE was observed, indicating a reduction in proton influx to the mitochondrial matrix. This observation points to a reduction in per- meability of the inner mitochondrial membrane to cations induced by DDE, suggesting a tighter lipid bilayer. Indeed, DDE-treated liposomes and native membranes showed an increased ordering in membranes with low cholesterol content [33]. Given that the inner mitochondrial membrane possesses a small amount of cholesterol the reduction in testis mitochondria permeability may be due to an ordering effect. In the titration assay liver mitochondria showed an increase in proton influx through the inner mitochondrial membrane as described previously [12]. Because liver mitochondria did not show significant differences in passive proton leak, we can speculate that the proton leak observed in energized mitochondria, is not only due to the movement of $\mathrm{H}^{+}$through the lipid bilayer but also most likely mediated by proteins/channels regulated by electrical potential such as uncoupling proteins (UCPs) or the adenine nucleotide translocase (ANT) (for review see [34]). In liver mitochondria the possible ordering effect detected in testis mitochondria may be masked by the increase in proton leak mediated by proteins or may be absent due to differences in lipid content of the inner mitochondrial membrane of both types of mitochondria. Indeed, small differences in inner mitochondrial membrane lipid composition, related to phospholipid/cholesterol ratios, are known to alter drugs effects [35].

Nevertheless, the reduced proton leak observed in nonenergized testis mitochondria does not totally explain the slight increase and maintenance of the electric potential in DDE treated mitochondria. Other factors such as inhibition of ATP-regulated $\mathrm{K}^{+}$ channels (suggested by ATP inhibition of the DDE effect on the increase in maximum developed potential - data not shown) may counteract the decrease in electrical potential due to the inhibition of the redox chain.

In conclusion, after showing that testicular mitochondria bioenergetics are distinct from more commonly used liver mitochondria $[13,14]$, we show here that this can have consequences in terms of inferring substance toxicity. In fact DDE has a clear signature effect on the oxidative phosphorylation system of testicular mitochondria. The changes detected may be responsible for part of the reproductive pathology induced by DDE, namely the recorded effects in apoptosis in both cultured Sertoli cells and in vivo germ cells [8-10], where links to mitochondrial dysfunction were reported. These effects should therefore be analyzed as a complement to the established endocrine disruptor effects of this environmental contaminant. Another major conclusion of this study is the possible misuse of liver mitochondria when inferring possible toxic effects of drugs and contaminants on the reproductive system. Whenever possible, testis and/or sperm mitochondria should be used instead.

\section{Conflict of interest statement}

None.

\section{Acknowledgements}

The authors would like to thank Dr. Sancha Santos the unconditional lab support. We would also like to thank Ana Paula Sousa for help with animal treatment and Gonçalo Pereira for assistance in protocol development for testicular mitochondria. Paula Mota was supported by a FCT PhD Fellowship (Portugal).

\section{References}

[1] Rignell-Hydbom A, Rylander L, Giwercman A, Jönsson BA, Lindh C, Eleuteri P, et al. Exposure to PCBs and p,p'-DDE and human sperm chromatin integrity. Environ Health Perspect 2005;113:175-9.

[2] Saradha B, Mathur PP. Effect of environmental contaminants on male reproduction. Environ Toxicol Pharmacol 2006;21:34-41. 
[3] Anway MD, Skinner MK. Epigenetic transgenerational actions of endocrine disruptors. Endocrinology 2006;147(Suppl.):43-9.

[4] WHO (World Health Organization). DDT and its derivatives in drinking-water. In: Background document for development of WHO guidelines for drinkingwater quality. World Health Organization; 2004. pp. 1-7.

[5] Lundholm CD. DDE-induced eggshell thinning in birds: effects of p,p'-DDE on the calcium and prostaglandin metabolism of the eggshell gland. Comp Biochem Physiol C: Pharmacol Toxicol Endocrinol 1997;118:113-28.

[6] Vonier PM, Crain DA, McLachlan JA, Guillette Jr LJ, Arnold SF. Interaction of environmental chemicals with the estrogen and progesterone receptors from the oviduct of the American alligator. Environ Health Perspect 1996;104:1318-22.

[7] Kelce WR, Stone CR, Laws SC, Gray LE, Kemppainen JA, Wilson EM. Persistent DDT metabolite p, $\mathrm{p}^{\prime}$-DDE is a potent androgen receptor antagonist. Nature 1995;375:581-5.

[8] Xiong X, Wang A, Liu G, Liu H, Wang C, Xia T, et al. Effects of p,p'dichlorodiphenyldichloroethylene on the expressions of transferrin and androgen-binding protein in rat Sertoli cells. Environ Res 2006;101:334-9.

[9] Song Y, Liang X, Hu Y, Wang Y, Yu H, Yang K. p,p'-DDE induces mitochondriamediated apoptosis of cultured rat Sertoli cells. Toxicology 2008:253:53-61.

[10] Shi YQ, Wang YP, Song Y, Li HW, Liu CJ, Wu ZG, et al. p,p'-DDE induces testicular apoptosis in prepubertal rats via the Fas/FasL pathway. Toxicol Lett 2010;193:79-85.

[11] Pereira SP, Pereira GC, Moreno AJ, Oliveira PJ. Can drug safety be predicted and animal experiments reduced by using isolated mitochondrial fractions? Altern Lab Anim 2009;37:355-65.

[12] Ferreira FM, Madeira VM, Moreno AJ. Interactions of 2,2-bis(p-chlorophenyl)1,1-dichloroethylene with mitochondrial oxidative phosphorylation. Biochem Pharmacol 1997;53:299-308.

[13] Amaral S, Mota PC, Lacerda B, Alves M, Pereira Mde L, Oliveira PJ, et al. Testicular mitochondrial alterations in untreated streptozotocin-induced diabetic rats. Mitochondrion 2009;9:41-50.

[14] Mota P, Amaral S, Martins L, de Lourdes Pereira M, Oliveira PJ, Ramalho-Santos J. Mitochondrial bioenergetics of testicular cells from the domestic cat (Felis catus)-a model for endangered species. Reprod Toxicol 2009;27:111-6.

[15] Moreno AJ, Oliveira PJ, Nova CD, Alvaro AR, Moreira RA, Santos SM, et al. Unaltered hepatic oxidative phosphorylation and mitochondrial permeability transition in wistar rats treated with nimesulide: relevance for nimesulide toxicity characterization. J Biochem Mol Toxicol 2007;21:53-61.

[16] Kamo N, Muratsugu M, Hongoh R, Kobatake Y. Membrane potential of mitochondria measured with an electrode sensitive to tetraphenyl phosphonium and relationship between proton electrochemical potential and phosphorylation potential in steady state. J Membr Biol 1979;49:105-21.

[17] Madeira VM, Antunes-Madeira MC, Carvalho AP. Activation energies of the ATPase activity of sarcoplasmic reticulum. Biochem Biophys Res Commun 1974;58:897-904.

[18] Estabrook RE. Mitochondrial respiratory control and the polarographic measurement of ADP/O ratios. Methods Enzymol 1967;10:41-7.

[19] Chance B, Williams GR. The respiratory chain and oxidative phosphorylation. Adv Enzymol Relat Subj Biochem 1956;17:65-134.
[20] Moreira PI, Santos MS, Moreno AM, Proença T, Seiça R, Oliveira CR. Effect of streptozotocin-induced diabetes on rat brain mitochondria. J Neuroendocrinol 2004; $16: 32-8$.

[21] Rodrigues AS, Lacerda B, Moreno AJ, Ramalho-Santos J. Proton leak modulation in testicular mitochondria affects reactive oxygen species production and lipid peroxidation. Cell Biochem Funct 2010;28:224-31.

[22] Oliveira PJ, Rolo AP, Sardão VA, Coxito PM, Palmeira CM, Moreno AJ. Carvedilol in heart mitochondria: protonophore or opener of the mitochondrial K(ATP) channels. Life Sci 2001;69:123-32.

[23] Masini A, Ceccarelli-Stanzani D, Muscatello U. An investigation on the effect of oligomycin on state-4 respiration in isolated rat-liver mitochondria. Biochim Biophys Acta 1984;767:130-7.

[24] Du G, Mouthys-Mickalad A, Sluse F. Generation of superoxide anion by mitochondria and imparment of their functions during anoxia and reoxygenation in vitro. Free Radic Biol Med 1998;25:1066-74.

[25] Bernanke J, Köhler HR. The impact of environmental chemicals on wildlife vertebrates. Rev Environ Contam Toxicol 2009;198:1-47.

[26] Amaral S, Mota P, Rodrigues AS, Martins L, Oliveira PJ, Ramalho-Santos J. Testicular aging involves mitochondrial dysfunction as well as an increase in UCP2 levels and proton leak. FEBS Lett 2008;582:4191-6.

[27] Alleva E, Francia N, Pandolfi M, De Marinis AM, Chiarotti F, Santucci D. Organochlorine and heavy-metal contaminants in wild mammals and birds of Urbino-Pesaro Province Italy: an analytic overview for potential bioindicators. Arch Environ Contam Toxicol 2006;51:123-34.

[28] Moreno AJ, Madeira VM. Mitochondrial bioenergetics as affected by DDT. Biochim Biophys Acta 1991;1060:166-74.

[29] Korshunov SS, Skulachev VP, Starkov AA. High protonic potential actuates a mechanism of production of reactive oxygen species in mitochondria. FEBS Lett 1997;416:15-8.

[30] de Jager C, Aneck-Hahn NH, Bornman MS, Farias P, Leter G, Eleuteri P, et al. Sperm chromatin integrity in DDT-exposed young men living in a malaria area in the Limpopo Province, South Africa. Hum Reprod 2009;24:242938.

[31] De Jager C, Farias P, Barraza-Villarreal A, Avila MH, Ayotte P, Dewailly E, et al. Reduced seminal parameters associated with environmental DDT exposure and p,p'-DDE concentrations in men in Chiapas, Mexico: a cross-sectional study. J Androl 2006;27:16-27.

[32] Messaros BM, Rossano MG, Liu G, Diamond MP, Friderici K, Nummy-Jernigan K, et al. Negative effects of serum p, p'-DDE on sperm parameters and modification by genetic polymorphisms. Environ Res 2009;109:457-64.

[33] Antunes-Madeira Mdo C, Madeira VM. Effects of DDE on the fluidity of model and native membranes: implications for the mechanisms of toxicity. Biochim Biophys Acta 1993;1149:86-92.

[34] Jastroch M, Divakaruni AS, Mookerjee S, Treberg JR, Brand MD. Mitochondrial proton and electron leaks. Essays Biochem 2010;47:53-67.

[35] Woldegiorgis G, Shrago E. Adenine nucleotide translocase activity and sensitivity to inhibitors in hepatomas Comparison of the ADP/ATP carrier in mitochondria and in a purified reconstituted liposome system. J Biol Chem 1985;260:7585-90. 